
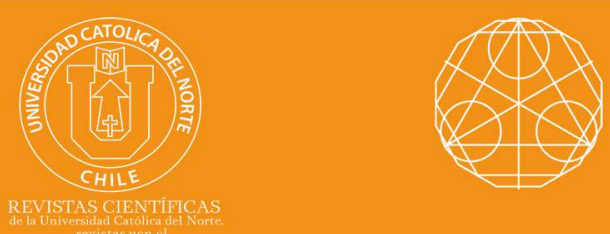

\title{
Nonlocal quantum stochastic differential equations with impulsive effects
}

\author{
M. O. Ogundiran* (iD) orcid.org/0000-0003-1210-7385
}

*Obafemi Awolowo University, Dept. of Mathematics, Ile-Ife, Nigeria.

Vmogundiran@oauife.edu.ng

\section{Received: October 2018 | Accepted: March 2019}

\section{Abstract:}

The aim of this work is to establish further existence of solution results for quantum stochastic differential equations with unbounded coefficients, which are also stochastic processes. Existence of solutions for nonlocal quantum stochastic differential equations with impulsive effects is established. The nonlocal condition extends the traditional initial value condition and cases of Lipschitz and non-Lipschitz continuous conditions were established. The quantum stochastic differential equation considered are driven by noises on Boson Fock spaces and measure of non compactness was employed to prove the main result.

Keywords: Quantum stochastic processes; Impulse effects; Fixed points.

MSC (2010): 34A37, 81S25.

\section{Cite this article as (IEEE citation style):}

S. Ulagammal, "Nonlocal quantum stochastic differential equations with impulsive effects", Proyecciones (Antofagasta, On line), vol. 38, no. 5, pp. 1057-1069, Dec. 2019, doi: 10.22199/issn.0717-62792019-05-0068. [Accessed dd-mm-yyyy].

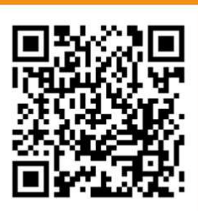

Article copyright: (c) 2019 M. 0. Ogundiran. This is an open access article distributed under the terms of the Creative Commons Licence, which permits unrestricted use and distribution provided the original author and source are credited. 


\section{Introduction}

The theory of impulsive differential equations has attracted the interest of many authors because of its vast applications in various fields. The impulsive conditions describe some systems much better than just initial conditions especially when the evolutions of such systems are prone to rapid change at certain moments. The study of impulsive differential equations has been done in [1], [2], [4], [11] and the references cited in them for classical differential equations and inclusions. In a like manner, the existence and uniqueness of solution for impulsive quantum stochastic differential equations was established in [13] as an extension of Hudson-Pathasarathy quantum stochastic differential equation [12]. Existence of solutions of non commutative stochastic differential equations in different locally convex spaces were established in [8]. The controllability of quantum stochastic differential inclusions was established in [14]. In [5], the techniques of fixed point theory to quantum stochastic differential equations were done for the first time. Moreover, the existence of solutions for quantum stochastic differential equations with unbounded coefficients was established in [9].

Nonlocal Cauchy problem has more applications than classical initial value problem. A study of semilinear initial value problems with nonlocal conditions was done in [6]. Many authors have since been attracted to problems with such nonlocal conditions [16] but of interest to us is the case in which the infinitesimal generator of the semigroups involved in the system is not necessarily compact. To circumvent such situation, the technique of measure of non compactness was used [3], [7].

The motivation for works on quantum stochastic differential equations stems from their applications in quantum optics, quantum measurements, quantum information to mention a few. Also, the dynamics of such systems described by quantum stochastic differential equations are well studied by various properties of solutions of the equation.

The aim of this work is to adapt the measure of noncompactness technique establish the existence of solution to noncommutative stochastic differential equations. This will not only extend results in literatures on quantum stochastic differential equations with unbounded coefficients but will enhance further applications to quantum stochastic flows generated by such solutions. Moreover, the work gives a generalization of such results with 
non local condition on classical ordinary differential equations to the present non commutative settings.

\section{Preliminaries}

In this subsection we shall introduce the notations and definitions on Quantum stochastic differential equations as it is done in [8] and it is consistent with [13].

\subsection{Notations and Definitions}

Let $\mathbf{D}$ be some pre-Hilbert space whose completion is $\mathcal{R} ; \gamma$ is a fixed Hilbert; $L_{\gamma}^{2}\left(\mathbf{R}_{+}\right)$is the space of square integrable $\gamma$-valued maps on $\mathbf{R}_{+}$ and $\Gamma\left(L_{\gamma}^{2}\left(\mathbf{R}_{+}\right)\right)$is the Boson Fock space. In the sequel, $\underline{\otimes}$ denotes the algebraic tensor products while $\otimes$ denotes (topological) tensor products.

The inner product of the Hilbert space $\mathcal{R} \otimes \Gamma\left(L_{\gamma}^{2}\left(\mathbf{R}_{+}\right)\right)$will be denoted by $\langle.,$.$\rangle and \|$. $\|$ the norm induced by $\langle.,$.$\rangle . Let \mathbf{E}, \mathbf{E}_{t}$ and $\mathbf{E}^{t}, t>$ 0 be linear spaces generated by the exponential vectors in Fock spaces $\Gamma\left(L_{\gamma}^{2}\left(\mathbf{R}_{+}\right)\right), \Gamma\left(L_{\gamma}^{2}([0, t))\right)$ and $\Gamma\left(L_{\gamma}^{2}([t, \infty))\right)$ respectively ;

$$
\begin{gathered}
\mathcal{A} \equiv L_{w}^{+}\left(\mathbf{D} \underline{\otimes} \mathbf{E}, \mathcal{R} \otimes \Gamma\left(L_{\gamma}^{2}\left(\mathbf{R}_{+}\right)\right)\right) \\
\mathcal{A}_{t} \equiv L_{w}^{+}\left(\mathbf{D} \underline{\otimes} \mathbf{E}_{t}, \mathcal{R} \otimes \Gamma\left(L_{\gamma}^{2}([0, t))\right)\right) \otimes \mathbf{I}^{t} \\
\mathcal{A}^{t} \equiv \mathbf{I}_{t} \otimes L_{w}^{+}\left(\mathbf{E}^{t}, \Gamma\left(L_{\gamma}^{2}([t, \infty))\right)\right), t>0
\end{gathered}
$$

where $\underline{\otimes}$ denotes algebraic tensor product and $\mathbf{I}_{t}\left(\right.$ resp.. $\left.\mathbf{I}^{t}\right)$ denotes the identity map on $\left.\mathcal{R} \otimes \Gamma\left(L_{\gamma}^{2}([0, t))\right)\right)\left(\operatorname{resp} . \Gamma\left(L_{\gamma}^{2}([t, \infty))\right)\right), t>0$.

We define the locally convex space $\mathcal{A}$ of noncommutative stochastic processes whose topology $\tau_{w}$, is generated by the family of seminorms $\left\{\|x\|_{\eta \xi}=|\langle\eta, x \xi\rangle|, x \in \mathcal{A}, \eta, \xi \in \mathbf{D} \underline{\otimes} \mathbf{E}\right\}$. The completion of $\left(\mathcal{A}, \tau_{w}\right)$ (resp. $\left.\left(\mathcal{A}_{t}, \tau_{w}\right)\right)$ is denoted by $\widetilde{\mathcal{A}}$ (resp. $\left.\widetilde{\mathcal{A}_{t}}\right)$ The underlying elements of $\widetilde{\mathcal{A}}$ consist of linear maps from $\mathbf{D} \otimes \mathbf{E}$ into $\mathcal{R} \otimes \Gamma\left(L_{\gamma}^{2}\left(\mathbf{R}_{+}\right)\right)$having domains of their adjoints containing $\mathbf{D} \otimes \mathbf{E}$. For a fixed Hilbert space $\gamma$, the spaces $L_{l o c}^{p}(\widetilde{\mathcal{A}})$, $L_{\gamma, l o c}^{\infty}\left(\mathbf{R}_{+}\right)$and $L_{l o c}^{p}(I \times \widetilde{\mathcal{A}})$ are adopted as in [8].

By a stochastic process indexed by $I=[0, T] \subseteq \mathbf{R}_{+}$, we mean a function on $I$ with values in $\operatorname{clos}(\widetilde{\mathcal{A}})$. 
A stochastic process $\Phi$ will be called (i) adapted if $\Phi(t) \subseteq \widetilde{\mathcal{A}}_{t}$ for each $t \in \mathbf{R}_{+}$; (ii) measurable if $t \mapsto d_{\eta \xi}(x, \Phi(t)$ ) is measurable for arbitrary $x \in \widetilde{\mathcal{A}}, \eta, \xi \in \mathbf{D} \otimes \mathbf{E}$; (iii) locally absolutely $p$ integrable if $t \mapsto\|\Phi(t)\|_{\eta \xi}, t \in \mathbf{R}_{+}$, lies in $L_{l o c}^{p}(\widetilde{\mathcal{A}})$ for arbitrary $\eta, \xi \in \mathbf{D} \underline{\otimes} \mathbf{E}$

The set of all absolutely $p$-integrable stochastic processes will be denoted by $L_{l o c}^{p}(\widetilde{\mathcal{A}})$ and for $p \in(0, \infty), L_{l o c}^{p}(I \times \widetilde{\mathcal{A}})$ is the set of maps $\Phi: I \times \widetilde{\mathcal{A}} \rightarrow \operatorname{clos}(\widetilde{\mathcal{A}})$ such that $t \mapsto \Phi(t, X(t)), t \in I$ lies in $L_{l o c}^{p}(\widetilde{\mathcal{A}})_{m v s}$ for every $X \in L_{l o c}^{p}(\widetilde{\mathcal{A}})$.

The Boson stochastic integrators; annihilation, creation and conservation processes arising from quantum field operators as introduced in [12] gave rise to quantum stochastic differential equation. We shall employ this formulation in the sequel.

Consider stochastic processes $E, F, G, H \in L_{l o c}^{2}(I \times \widetilde{\mathcal{A}})$ and $\left(0, x_{0}\right)$ be a fixed point in $[0, T] \times \widetilde{\mathcal{A}}$. Then, a relation of the form

$$
\begin{gathered}
X(t)=x_{0}+\int_{0}^{t}\left(E(s, X(s)) d \Lambda_{\pi}(s)+F(s, X(s)) d A_{f}(s)\right. \\
\left.+G(s, X(s)) d A_{g}^{+}(s)+H(s, X(s)) d s \quad t \in[0, T]\right)
\end{gathered}
$$

will be called a stochastic integral equation with coefficients $E, F, G$ and $H$.

The stochastic differential equation corresponding to the integral equation above is;

$$
\begin{aligned}
d X(t) & =E(t, X(t)) d \Lambda_{\pi}(t)+F(t, X(t)) d A_{f}(t) \\
& +G(t, X(t)) d A_{g}^{+}(t)+H(t, X(t)) d t \\
X(0) \quad & =x_{0} \quad \text { almost all } t \in[0, T]
\end{aligned}
$$

The notion of solution of (2.1) is defined as follows:

Definition 2.1. Let $A d(\widetilde{\mathcal{A}})_{\text {wac }}$ denotes the set of all adapted weakly absolutely continuous stochastic processes on $\widetilde{\mathcal{A}}$. By a solution of (2.1)we mean a stochastic process $\varphi \in \operatorname{Ad}(\widetilde{\mathcal{A}})_{\text {wac }} \cap L_{\text {loc }}^{2}(\widetilde{\mathcal{A}})$ such that

$$
\begin{aligned}
d \varphi(t) & =E(t, \varphi(t)) d \Lambda_{\pi}(t)+F(t, \varphi(t)) d A_{f}(t) \\
& +G(t, \varphi(t)) d A_{g}^{+}(t)+H(t, \varphi(t)) d t \text { almost all } t \in I \\
\varphi\left(t_{0}\right) & =\varphi_{0}
\end{aligned}
$$


This work shall be concerned with the existence of solution of

$$
\begin{array}{ll}
d x(t) & =A x(t) d t+E(t, x(t)) d \Lambda_{\pi}(t)+F(t, x(t)) d A_{f}(t) \\
& +G(t, x(t)) d A_{g}^{+}(t)+H(t, x(t)) d t \$ \text { almostall } \quad \mathrm{t} \in[0, T] \\
x(0) \quad & =g(x) \\
\Delta x\left(t_{i}\right) & =I_{i}\left(x\left(t_{i}\right)\right) \quad i=1,2, \ldots, p ; \quad 0<t_{1}<t_{2}<\ldots<t_{p}<T
\end{array}
$$

where $A: D(A) \subset \widetilde{\mathcal{A}} \rightarrow \widetilde{\mathcal{A}}$ is a non-densely defined operator, $x\left(t_{i}^{+}\right), x\left(t_{i}^{-}\right)$ denotes the right and the left limits of $x$ at $t_{i}$ respectively and $\Delta x\left(t_{i}\right)=$ $x\left(t_{i}^{+}\right)-x\left(t_{i}^{-}\right) . I_{i}$ denotes the impulsive effects at $t_{i}$.

$P C([0, T], \widetilde{\mathcal{A}})=\left\{x:[0, T] \rightarrow \widetilde{\mathcal{A}}: x(t)\right.$ is a continuous adapted stochastic process at $t \neq t_{i}$ left continuous at $t=t_{i}$ and the right limit $x\left(t_{i}\right)$ exists for $\left.i=1,2, \ldots, p\right\}$

equip with the norm

$$
\|x\|_{P C}=\sup \{|\langle\eta, x(t) \xi\rangle|:, x \in \widetilde{\mathcal{A}}, \eta, \xi \in \mathbf{D} \underline{\otimes} \mathbf{E}, t \in[0, T]\}
$$

(where the supremum is taken over some bounded subsets of $\mathbf{D} \underline{\otimes} \mathbf{E}$ ) is a Banach space which shall be denoted by $P C$.

Let $r$ be finite positive constant. We consider the sets $B_{r}=\{x \in \widetilde{\mathcal{A}}: \|$ $\left.x \|_{\eta \xi} \leq r\right\}, W_{r}=\left\{x \in P C: x(t) \in B_{r}, \forall t \in[0, T]\right\}$.

An operator-valued stochastic process $x:[0, T] \rightarrow \widetilde{\mathcal{A}}$ is said to be an integral solution of $(2.2)$ on $[0, T]$ if the following conditions hold;

(i) $X \in P C ;($ ii $) \int_{0}^{s} X(s) d s \in D(A)$ for $t \in[0, T]$ and

(iii)X(t) $=g(X)+A \int_{0}^{t} X(s) d s+\int_{0}^{t}\left(E(s, X(s)) d \Lambda_{\pi}(s)+F(s, X(s)) d A_{f}(s)\right.$

$$
+G(s, X(s)) d A_{g}^{+}(s)+H(s, X(s)) d s+\sum_{0<t_{i}<t} I_{i}\left(X\left(t_{i}\right)\right) \text { for } t \in[0, T]
$$

Hausdorff measure of non-compactness $\alpha($.$) is defined on each bounded$ subset $\Omega$ of a Banach space $Y$ by

$$
\alpha(\Omega)=\inf \{\epsilon>0: \Omega \text { has a finite } \epsilon-\text { net in } Y\}
$$

some basic properties of $\alpha($.$) are given in the following Lemma.$

Lemma 2.1. ([3]). Let $Y$ be a real Banach space and $B, C \subseteq Y$ be bounded, the following properties are satisfied:

(1) $B$ is pre-compact if and only if $\alpha(B)=0$;

(2) $\alpha(B)=\alpha(\bar{B})=\alpha(\operatorname{conv} B)$, where $\bar{B}$ and conv $B$ mean the closure and convex hull of $B$, respectively

(3) $\alpha(B) \leq \alpha(C)$ when $B \subseteq C$

(4) $\alpha(B+C) \leq \alpha(B)+\alpha(C)$, where $B+C=\{x+y ; x \in B, y \in C\}$; 
(5) $\alpha(B \cup C)=\max \{\alpha(B), \alpha(C)\}$

(6) $\alpha(\lambda B)=|\lambda| \alpha(B)$ for any $\lambda \in \mathbf{R}$

(7) If the map $Q: D(Q) \subseteq Y \rightarrow Z$ is Lipschitz continuous with constant $k$, then $\alpha(Q B) \leq k \alpha(B)$ for any bounded subset $B \subseteq D(Q)$, where $Z$ is a Banach spice.

The map $Q: W \subseteq Y \rightarrow Y$ is said to be an $\alpha$-contraction if there exists a positive constant $k<1$ such that $\alpha(Q C) \leq k \alpha(C)$ for any bounded closed subset $C \subseteq W$, where $Y$ is a Banach space.

Lemma 2.2. ([3], Darbo-Sadovskii). If $W \subseteq Y$ is bounded closed and convex, the continuous map $Q: W \rightarrow W$ is an $\alpha$-contraction, then the map $Q$ has at least one fixed point in $W$.

The linear operator $A: D(A) \subset \widetilde{\mathcal{A}} \rightarrow \widetilde{\mathcal{A}}$ is said to satisfy the HilleYosida condition if there exists $K \geq 0$ and $\omega \in \mathbf{R}$ such that $(\omega,+\infty) \subset \rho(A)$ and

$$
\sup \left\{(\lambda-\omega)^{n}\left\|R(\lambda, A)^{n}\right\|_{\eta \xi} ; n \in \mathbf{N}, \lambda>\omega\right\} \leq K
$$

where $R(\lambda, A)=(\lambda I-A)^{-1}, \rho(A)$ is the resolvent set of $A$.

Let $A_{0}$ be the part of $A$ in $\overline{D(A)}$ defined by

$$
\begin{aligned}
& D\left(A_{0}\right)=\{x \in D(A): A x \in \overline{D(A)}\}, \\
& A_{0} x=A x .
\end{aligned}
$$

Then $A_{0}$ generates a $C_{0}$-semigroup $\{S(t)\}_{t \geq 0}$ on $\overline{D(A)}$ and if the integral solution exists as above then it is given by

$$
\begin{aligned}
X(t) & =S(t) g(X)+\lim _{\lambda \rightarrow \infty} \int_{0}^{t} S(t-s) B(\lambda)\left(E(s, X(s)) d \Lambda_{\pi}(s)+F(s, X(s)) d A_{f}(s)\right. \\
& \left.+G(s, X(s)) d A_{g}^{+}(s)+H(s, X(s)) d s\right)+\sum_{0<t_{i}<t} S\left(t-t_{i}\right) I_{i}\left(X\left(t_{i}\right)\right) 0 \leq t \leq T .
\end{aligned}
$$

where $B(\lambda)=\lambda \mathbf{R}(\lambda ; A)$

The following hypotheses are employed in the sequel:

$H_{1}$ : The linear operator $A: D(A) \rightarrow \widetilde{\mathcal{A}}$ satisfies the Hille-Yosida condition. $H_{2}$ : The operator $S(t)$ generated by $A_{0}$ is compact in $\overline{D(A)}$ when $t>0$ and

$M=\sup _{t \in[0, T]}\|S(t)\|_{\eta \xi}, \eta, \xi \in \mathbf{D} \underline{\otimes} \mathbf{E}$.

$H_{3}$ : The operator $S(t), 0 \leq t \leq T$ generated by $A_{0}$ is equicontinuous.

$H_{4}:$ (i) $E, F, G, H:[0, T] \times \widetilde{\mathcal{A}} \rightarrow \widetilde{\mathcal{A}}$ are Carathéodory i.e. for $M=$ $\{E, F, G, H\}$

$M(t,):.[0, T] \times \widetilde{\mathcal{A}} \rightarrow \widetilde{\mathcal{A}}$ is continuous $\forall X \in \widetilde{\mathcal{A}}$ and $M(., X):[0, T] \times \widetilde{\mathcal{A}} \rightarrow \widetilde{\mathcal{A}}$ is measurable.

(ii) For any $l>0, \exists$ a function $\rho_{l} \in L^{1}([0, T], \mathbf{R})$ such that

$$
\|M(t, X)\|_{\eta \xi} \leq \rho_{l}(t) \text { for a.e.t } \in[0, T] \text { andall } X \in B_{l}
$$


Nonlocal quantum stochastic differential equations with impulsive.1063

(iii) There exists a function $m \in L^{1}([0, s], \mathbf{R})$ and a non-decreasing continuous function $\psi:[0, \infty) \rightarrow(0, \infty)$ such that

$$
\|M(t, X)\|_{\eta \xi} \leq m(t) \psi\left(\|X\|_{\eta \xi}\right) \quad \forall X \in \widetilde{\mathcal{A}}, t \in[0, T]
$$

$H_{5}$ : There exists a function $h \in L^{1}(0, s ; \mathbf{R})$ such that for every bounded $D \subseteq W_{r}$

$$
\alpha(M(t, D)) \leq h(t) \alpha(D) \text { fora.e.t } \in[0, T],
$$

where $\alpha$ is the Hausdorff measure of noncompactness.

$H_{6}: g: P C \rightarrow \overline{D(A)}$ is Lipschitz continuous with constant $k$;

$H_{7}: I_{i}: \widetilde{\mathcal{A}} \rightarrow \overline{D(A)}$ is Lipschitz continuous $k_{i}, i=1,2, \ldots, p$.

\section{Main Results}

We consider the cases when the nonlocal function $g$ are Lipschitz continuous and when they are not continuous.

\subsection{The case $\mathrm{g}$ is Lipschitz}

Theorem 3.1. Assume that the conditions $\left(H_{1}\right)-\left(H_{7}\right)$ are satisfied. Then the nonlocal Cauchy problem (2.2) has at least one integral solution on $[0, T]$ provided that

$$
M\left[\left(k+\sum_{i=1}^{p} k_{i}\right) r+\|g(0)\|+\sum_{i=1}^{p}\left\|I_{i}(0)\right\|+K\left\|\rho_{r}\right\|_{L^{1}}\right] \leq r
$$

Proof

We consider the operator $Q: P C \rightarrow P C$ defined by

$(Q x)(t)=\left(Q_{1} x\right)(t)+\left(Q_{2} x\right)(t)+\left(Q_{3} x\right)(t)$ where

$\left(Q_{1} x\right)(t)=S(t) g(x)$,

$\left(Q_{2} x\right)(t)=\lim _{\lambda \rightarrow \infty} \int_{0}^{t} S(t-s) B(\lambda)\left(E(s, x(s)) d \Lambda_{\pi}(s)+F(s, x(s)) d A_{f}(s)\right.$

$\left.+G(s, x(s)) d A_{g}^{+}(s)+H(s, x(s)) d s\right)$

$\left(Q_{3} x\right)(t)=\sum_{0<t_{i}<t} S\left(t-t_{i}\right) I_{i}\left(x\left(t_{i}\right)\right)$

for all $t \in[0, T]$ The fixed point of $Q$ is the integral solution of the nonlocal impulsive problem (2.2). We will prove that $Q$ has a fixed point by using Lemma 2.2.

Firstly, we will prove that the mapping $Q$ is continuous on PC. Let $\left\{x_{n}\right\}_{n=1}^{\infty}$ be a sequence in $\mathrm{PC}$ with $\lim _{n \rightarrow \infty} x_{n}=x$ in PC. By the continuity of $E, F, G, H$ with respect to the second argument, we deduce that for each 
$s \in[0, T], M\left(s, x_{n}(s)\right)$ converges to $M(s, x(s))$ in $\widetilde{\mathcal{A}}, M \in\{E, F, G, H\}$ and we have

$$
\begin{aligned}
\left\|Q x_{n}-Q x\right\|_{P C} & \leq M\left[\left\|g\left(x_{n}\right)-g(x)\right\|+\sum_{i=1}^{p}\left\|I_{i}\left(x_{n}\left(t_{i}\right)\right)-I_{i}\left(x\left(t_{i}\right)\right)\right\|\right] \\
& +M K \int_{0}^{T}\left(\left\|E\left(s, x_{n}(s)\right)-E(s, x(s))\right\| d \Lambda_{\pi}(s)\right. \\
& +\left\|F\left(s, x_{n}(s)\right)-F(s, x(s))\right\| d A_{f}(s) \\
& +\left\|G\left(s, x_{n}(s)\right)-G(s, x(s))\right\| d A_{g}^{+}(s) \\
& \left.\left.+\left\|H\left(s, x_{n}(s)\right)-H(s, x(s))\right\| d s\right)\right)
\end{aligned}
$$

Then by continuity of $g, I_{i}$ and using dominated convergence theorem, we get $\lim _{n \rightarrow \infty} Q u_{n}=Q u$ in $\mathrm{PC}$, which implies that the mapping $Q$ is continuous on PC.

Secondly, we claim that $Q W_{r} \subseteq W_{r}$. In fact, for any $x \in W_{r} \subseteq P C$, by $\left(H_{4}\right)(i i)$, and

$$
\|B(\lambda)\| \leq \frac{\lambda K}{\lambda-\omega} \rightarrow K, \text { as } \lambda \rightarrow+\infty
$$

We have

$$
\begin{aligned}
\|(Q x)(t)\| & \leq\|S(t) g(x)\|+\| \lim _{\lambda \rightarrow+\infty} \int_{0}^{t} S(t-s) B(\lambda)\left(E(s, x(s)) d \Lambda_{\pi}(s)+F(s, x(s)) d A_{f}(s)\right. \\
& \left.\left.+G(s, x(s)) d A_{g}^{+}(s)+H(s, x(s)) d s\right)\right)\|+\| \sum_{0<t_{i}<t} S\left(t-t_{i}\right) I_{i}\left(x\left(t_{i}\right)\right) \| \\
& \leq M[\|g(x)-g(0)\|+\|g(0)\|]+M K \int_{0}^{T} \|\left(E(s, x(s)) d \Lambda_{\pi}(s)+F(s, x(s)) d A_{f}(s)\right. \\
& \left.\left.+G(s, x(s)) d A_{g}^{+}(s)+H(s, x(s)) d s\right)\right) \| \\
& +M\left[\sum_{i=1}^{p}\left\|I_{i}\left(x\left(t_{i}\right)\right)-I_{i}(0)\right\|+\left\|I_{i}(0)\right\|\right] \\
& \leq M\left[\left(k+\sum_{i=1}^{p} k_{i}\right) r+\|g(0)\|+\sum_{i=1}^{p}\left\|I_{i}(0)\right\|+K\left\|\rho_{r}\right\|_{L^{1}}\right] \\
& \leq r
\end{aligned}
$$

It implies that $Q W_{r} \subseteq W_{r}$.

Now according to Lemma 2.2, it remains to prove that $Q$ is an $\alpha$ contraction in $W_{r}$. By using conditions $\left(H_{6}\right)$ and $\left(H_{7}\right)$, we get that $Q_{1}+Q_{2}$ : $W_{r} \rightarrow P C$ is Lipschitz continuous with constant $M\left(k+\sum_{i=1}^{p} k_{i}\right)$. In fact, for any $x, y \in W_{r}$, we have 


$$
\begin{aligned}
\left\|\left(Q_{1}+Q_{2}\right) x-\left(Q_{1}+Q_{2}\right) y\right\|_{P C} & \leq \sup _{t \in[0, T]}\left[\| S(t)[g(x)-g(y)]+\sum_{i=1}^{p} S\left(t-t_{i}\right)\left[I_{i}\left(x\left(t_{i}\right)\right)\right]-I_{i} y\left(t_{i}\right)\right. \\
& \leq M\left[k\|x-y\|_{P C}+\sum_{i=1}^{p} k_{i}\|x-y\|_{P C}\right] \\
& =M\left[k+\sum_{i=1}^{p} k_{i}\right]\|x-y\|_{P C}
\end{aligned}
$$

Thus, by Lemma 2.1 [3], we obtain that

$$
\alpha\left(\left(Q_{1}+Q_{2}\right) W_{r}\right) \leq M\left[k+\sum_{i=1}^{p} k_{i}\right] \alpha\left(W_{r}\right) .
$$

Finally, we prove that $Q_{3}: W_{r} \rightarrow P C$ is a compact operator by using Arzéla-Ascoli's theorem. Since $Q_{3}$ is compact, thus $\alpha\left(Q_{3} W_{r}\right)=0$. Consequently,

$$
\begin{aligned}
\alpha\left(Q W_{r}\right) & \leq \alpha\left(\left(Q_{1}+Q_{2}\right) W_{r}\right)+\alpha\left(Q_{3} W_{r}\right) \\
& \leq M\left[k+\sum_{i=1}^{p} k_{i}\right] \alpha\left(W_{r}\right)
\end{aligned}
$$

From the condition (3.1), $M\left[k+\sum_{i=1}^{p} k_{i}\right] r<r$ which implies that $M[k+$ $\left.\sum_{i=1}^{p} k_{i}\right]<1$, hence the mapping $Q$ is an $\alpha$-contraction in $W_{r}$. By DarboSadovskii's fixed point theorem, the operator $Q$ has a fixed point in $W_{r}$ which is the integral solution of the nonlocal impulsive problem (2.2).

\subsection{The case $\mathrm{g}$ is not Lipschitz}

In this section, we give the existence of integral solutions for the nonlocal impulsive problem (2.2) when the nonlocal function $g$ is not Lipschitz. We will still consider the sets $B_{r}, W_{r}$ in section 3.1 and the solution mapping $Q=Q_{1}+Q_{2}+Q_{3}$ as defined above.

Let $\overline{c o n v} B$ denotes the convex closed hull of set $B \subseteq P C$, we give the following assumptions which shall be employed in the result:

$\left(H_{8}\right): g: P C \rightarrow \widetilde{\mathcal{A}}$ is a continuous mapping;

$\left(H_{9}\right): I_{i}: \widetilde{\mathcal{A}} \rightarrow \widetilde{\mathcal{A}}$ is a continuous and compact mapping for every $i=$ $1,2, \ldots, p$

$\left(H_{10}\right)$ : The set $g\left(\operatorname{conv} Q W_{r}\right)$ is pre-compact.

Theorem 3.2. Assume that the conditions $\left(H_{1}\right)-\left(H_{5}\right),\left(H_{8}\right)-\left(H_{10}\right)$ are satisfied. Then the nonlocal impulsive problem (2.2) has at least one integral solution on $[0, T]$ provided that

$$
M\left[\sup _{x \in W_{r}}\|g(x)\|+\sup _{x \in W_{r}} \sum_{i=1}^{p}\left\|I_{i}\left(x\left(t_{i}\right)\right)\right\|+\left\|\rho_{r}\right\|_{L^{1}}\right] \leq r .
$$


Proof

From the continuity of $g, I_{i}$ and $E, F, G, H$; the mapping $Q$ is continuous on PC. We will use Schauder's fixed point theorem to obtain a fixed point of mapping $Q$.

Next, we claim that $Q W_{r} \subseteq W_{r}$. For any $x \in W_{r}$, by $\left(H_{4}\right)$ and (3.2), we have

$$
\begin{aligned}
\|(Q x)(t)\| & \leq\|S(t) g(x)\|+\sum_{0<t_{i}<t}\left\|S(t) I_{i}\left(x\left(t_{i}\right)\right)\right\|+\| \int_{0}^{t} S(t-s)\left(E(s, x(s)) d \Lambda_{\pi}(s)\right. \\
& \left.+F(s, x(s)) d A_{f}(s)+G(s, x(s)) d A_{g}^{+}(s)+H(s, x(s)) d s\right) \| \\
& \leq M\left[\sup _{x \in W_{r}}\|g(x)\|+\sup _{x \in W_{r}} \sum_{i=1}^{p}\left\|I_{i}\left(x\left(t_{i}\right)\right)\right\|+\left\|\rho_{r}\right\|_{L^{1}}\right] \\
& \leq r
\end{aligned}
$$

i.e. $Q$ maps $W_{r}$ into itself.

Lastly, by the hypotheses $\left(H_{1}\right)-\left(H_{3}\right)$ and $\left(H_{9}\right)$, the mappings $Q_{2}$ and $Q_{3}$ are compact in PC. That is, for every $t \in[0, T]$ and bounded set $W \subseteq P C, Q_{2} W(t)$ and $Q_{3} W(t)$ are relatively compact. $Q_{2} W(t)$ and $Q_{3} W(t)$ equicontinuous on $[0, T]$. Then, by the compactness $S(t), t>0$, we can deduce that $Q_{1} W_{r}(t)=S(t) g\left(W_{r}\right)$ is relatively compact for any $t \in(0, T]$ and $Q_{1} W_{r}(t)$ are equicontinuous on $(0, T]$.

Thus, for each $t \in(0, T], Q W_{r}(t)$ is relatively compact and $Q W_{r}$ is equicontinuous on $(0, T]$.

Let $W=\overline{c o n v} Q W_{r}$, we get that $W$ is a bounded closed and convex subset of $\mathrm{PC}$ and $Q W \subseteq W$. Moreover, we know that $g(W)=g\left(\overline{\operatorname{conv}} Q W_{r}\right)$ is pre-compact by condition $\left(H_{10}\right)$.

Now, for any $x \in W$ and $t \in\left(0, t_{i}\right)$, we have

$$
\left(Q_{2} x\right)(t)=\sum_{0<t_{1}<t} S\left(t-t_{i}\right) I_{i}\left(x\left(t_{i}\right)\right)=0 .
$$

It implies that

$$
\|Q x(t)-Q x(0)\| \leq\|(S(t)-I) g(x)\|+M \int_{0}^{t} \rho_{r}(s) d s .
$$

Since $g(W)$ is pre-compact, we have $\|Q x(t)-Q x(0)\| \rightarrow 0$ uniformly on $W$ as $t \rightarrow 0$. Thus, $Q W$ is equicontinuous at $t=0$ and $Q W(0)=g(W)$ is 
relatively compact, which implies that $Q: W \rightarrow W$ is a compact continuous operator. Therefore, by Schauder's fixed point theorem, we obtain a fixed point of mapping $Q$ on $W$, which is just the integral solution of the nonlocal impulsive problem (2.2).

\subsection{Example}

Let $\mathcal{R}$ be a system Hilbert space and $\Gamma\left(L^{2}\left(\mathbf{R}_{+}, \mathbf{C}\right)\right)$ the noise(or reservoir) Fock space, the interaction between the system and the reservoir is represented by the tensor product $\mathcal{R} \otimes \Gamma\left(L^{2}\left(\mathbf{R}_{+}, \mathbf{C}\right)\right)$. For a unitary operator $U_{t}$ (for each $t \geq 0$ ) defined on the Hilbert space $\mathcal{R} \otimes \Gamma\left(L^{2}\left(\mathbf{R}_{+}, \mathbf{C}\right)\right)$ the quantum stochastic flow

$$
j_{t}(X)=U_{t}^{*} X U_{t}
$$

satisfies the quantum stochastic differential equation ([12])

$$
\begin{aligned}
d j_{t}(X) & =j_{t}\left(i[H, X]-\frac{1}{2}\left(L^{*} L X+X L^{*} L-2 L^{*} X L\right)\right) d t \\
& +j_{t}\left(\left[L^{*}, X\right] W\right) d A_{t}+j_{t}\left(W^{*}[X, L]\right) d A_{t}^{+} \\
& +j_{t}\left(W^{*} X W-X\right) d \Lambda_{t} \\
j_{0}(X) & =X \\
\Delta j_{t}(X) & =I_{i}\left(j_{t_{i}}(X)\right)
\end{aligned}
$$

which is a generalization of classical quantum mechanical evolution equations.

It is an example of equation (2.2) where $X$ is a bounded system operator. The case of QSDE with unbounded coefficients was first considered in [10]. The unbounded property can be circumvented by the measure of non-compactness used above.

The added assumptions $\left(H_{8}\right),\left(H_{9}\right)$ and $\left(H_{10}\right)$ guaranteed the existence of unitary operator which satisfy such system and generalized the EvansHudson (E-H) flow [15] to the case with impulsive effects. In this case, the condition $\left(H_{10}\right)$ makes the unitary operator of the evolution to be relatively compact and not necessarily compact.

\section{Acknowledgements}

The author wishes to express his gratitude to International Mathematical Union(IMU) for the grant, IMU-SIMONS African Fellowship grants 2016, which gave him an opportunity for a Research visit to Volterra Centre of 
the University of Roma, Tor Vergata. He also appreciate Prof. L. Accardi who was his host and all members of staff of the centre for hospitality. This work was completed during the visit.

\section{References}

[1] A. Anguraj and M, Arjunan, "Existence and uniqueness of mild and classical solutions of impulsive evolution equations", Electronic journal of differential equations, vol. 2005, Art ID. 111, 2005. [On line]. Available: https://bit.ly/2PxpDJJ

[2] M. Arjunan, V. Kavitha and S. Selvi, "Existence results for impusive differential equations with nonlocal conditions via measures of noncompactness", Journal nonlinear sciences applications, vol. 5, no. 3, pp. 195205, 2012, doi: 10.22436/jnsa.005.03.04.

[3] J. Banas and K. Goebel, Measures of noncompactness in Banach spaces. New York, NY: Marcel Dekker, 1980.

[4] M. Benchohra, J. Henderson, and S. Ntouyas, Impulsive differential equations and inclusions, vol. 2. New York, NY: Hindawi Publishing Corporation, 2006.

[5] A. Boukas, "An application of fixed point theory to quantum SD", in Quantum probability and related topics, vol. 7, L. Accardi , Ed. World Scientific Publishing, 1993, pp. 95-97, doi: 10.1142/9789812812810_0008.

[6] L. Byszewski and V. Lakshmikantham, "Theorem about the existence and uniqueness of a solution of a nonlocal abstract cauchy problem in a banach space", Applicable analysis. An international journal, vol. 40, no. 1, pp. 11-19, 1991, doi: 10.1080/00036819008839989.

[7] K. Deimling, Multivalued differential equations. Berlin: Walter de Gruyter, 1992.

[8] G. Ekhaguere, "Topological solutions of noncommutative stochastic differential equations", Journal stochastic analysis and applications, vol. 25, no. 5, pp. 961-993, Aug. 2007, doi: 10.1080/07362990701540345.

[9] F. Fagnola, "On quantum stochastic differential equations with unbounded coefficients", Probability theory and related fields, vol. 86, no. 4, pp. 501-516, Dec. 1990, doi: 10.1007/BF01198172. 
[10] F. Fagnola and S. Wills, "Solving quantum stochastic differential equations with unbounded coefficients", Journal of functional analysis, vol. 198, no. 2, pp. 279- 310, Mar. 2003, doi: 10.1016/S00221236(02)00089-7.

[11] Z. Fan, "Impulsive problems for semilinear differential equations with nonlocal conditions", Nonlinear analysis: theory, methods \& applications, vol. 72, no. 2, pp. 1104-1109, Jan. 2010, doi: 10.1016/j.na.2009.07.049.

[12] R. Hudson and K. Parthasarathy, "Quantum Ito's formula and stochastic evolutions", Communications in mathematical physics, vol. 93, no. 3, pp. 301-323, Sep. 1984, doi: 10.1007/BF01258530.

[13] M. Ogundiran and V. Payne, "On the existence of solutions of impulsive quantum stochastic differential equations", Journal of differential equations and control processes, vol. 2013, no. 2, pp. 62-73, 2013. [On line]. Available: https://bit.ly/34zi200

[14] M. Ogundiran, "Controllability of quantum stochastic differential inclusions", Journal of nigerian mathematical society, vol. 35, no. 2, pp. 369380, 2016. [On line]. Available: https://bit.ly/2MkYdVt

[15] K. Parthasarathy, An introduction to quantum stochastic calculus. Basel: Birkhauser, 1992.

[16] X. Xue, "Existence of semilinear differential equations with nonlocal initial conditions", Acta mathematica sinica, english series, vol. 23, no. 6, pp. 983-988, Sep. 2007, doi: 10.1007/s10114-005-0839-3. 